\title{
Comunidad de Helmintos Parásitos del Sapo Espinoso Rhinella spinulosa (Wiegmann, 1834) (Anura: Bufonidae) de Perú
}

\author{
Community of Helminth Parasites of Thorny Toad Rhinella spinulosa (Anura: \\ Bufonidae) of Peru \\ Jhon Chero ${ }^{1,2}$, Celso Cruces $^{1,2}$, José Iannacone ${ }^{2,3}$, Gloria Sáez ${ }^{1}$, Lorena Alvariño ${ }^{2}$, \\ José Luque ${ }^{4}$, Víctor Morales ${ }^{3}$
}

\section{Resumen}

\begin{abstract}
El objetivo de la presente investigación fue evaluar la comunidad de los metazoos parásitos del sapo espinoso Rhinella spinulosa de Perú y elucidar si el sexo y la longitud total de los anfibios hospederos están estructurando sus comunidades de parásitos. Se trabajó con 90 especímenes de $R$. spinulosa adquiridos entre mayo de 2009 y octubre de 2010. La cavidad corporal, tubo digestivo, vejiga urinaria, pulmones y musculatura fueron examinados para la búsqueda de metazoos parásitos. Siete taxas de metazoos parásitos fueron colectados: un digeneo [Gorgoderina chilensis (Gorgoderidae), en vejiga urinaria], un cestodo [Cylindrotaenia aff. americana (Proteocephalidae) en intestino delgado], cuatro nematodos [Aplectana hylambatis y A. vellardi (Cosmocercidae) en intestino grueso, Hedruris moniezi (Hedruridae) en estómago y Rhabdias aff. pseudosphaerocephala (Rhabdiasidae) en pulmones] y un acantocefalo [Pseudoacanthocephalus lutzi (Echinorhynchidae) en intestino delgado]. Se observó una correlación positiva entre la talla y la abundancia media de $R$. aff. pseudosphaerocephala, así como asociación entre el sexo y la abundancia media de $G$. chilensis, A. hylambatis y R. aff. pseudosphaerocephala. G. chilensis mostró dependencia con el sexo de $R$. spinulosa. Los tres índices de agregación (Índice de Dispersión, Índice de Discrepancia de Poulin y K de la ecuación binomial negativa) indicaron una distribución contagiosa. La mayoría de los valores de diversidad alfa de las infracomunidades parasitarias fueron menores que los del componente comunitario.
\end{abstract}

Palabras clave: anfibio, anura, Aplectana, Gorgoderina, helminto, parásito, Perú, Rhabdias

\footnotetext{
${ }^{1}$ Laboratorio de Parasitología, ${ }^{2}$ Laboratorio de Ecología y Biodiversidad Animal (LEBA), Facultad de Ciencias Naturales y Matemática (FCNNM). Universidad Nacional Federico Villarreal (UNFV), Lima, Perú

${ }^{3}$ Facultad de Ciencias Biológicas, Universidad Ricardo Palma (URP), Lima, Perú

${ }^{4}$ Departamento de Parasitologia Animal, Universidade Federal Rural do Rio de Janeiro, RJ, Brazil
}

Recibido: 27 de julio de 2015

Aceptado para publicación: 14 de octubre de 2015 
The aim of this study was to evaluate the community of metazoan parasites of thorny toad Rhinella spinulosa of Peru and elucidate whether sex and the total length of the hosts amphibians are structuring their communities of parasites. Ninety specimens of $R$. spinulosa were acquired during May 2009 to October 2010. The body cavity, gastrointestinal tract, bladder, lungs and muscles were examined to search for metazoan parasites. Seven taxa of metazoan parasites were collected: one digenean [Gorgoderina chilensis (Gorgoderidae) in the bladder] a cestode [Cylindrotaenia aff. americana (Proteocephalidae) in the small intestine], four nematodes [Aplectana hylambatis and $A$. vellardi (Cosmocercidae) in the large intestine, Hedruris moniezi (Hedruridae) in the stomach and Rhabdias aff. pseudosphaerocephala (Rhabdiasidae) in lungs] and one acanthocephalan [Pseudoacanthocephalus lutzi (Echinorhynchidae) in the small intestine]. A positive correlation was observed between length and mean abundance of $R$. aff. pseudosphaerocephala, and an association between sex and mean abundance of G. chilensis, A. hylambatis and R. aff. pseudosphaerocephala. G. chilensis showed dependence on the sex of $R$. spinulosa. The three levels of aggregation (dispersion index, index discrepancy of Poulin and $\mathrm{K}$ negative binomial equation) showed a contagious distribution. Most of the values of alpha diversity of parasitic infracommunities were lower than those of the community component.

Key words: amphibian, anura, Aplectana, Gorgoderina, helminth, parasite, Peru, Rhabdias

\section{INTRODUCCIÓN}

Las comunidades de macroparásitos de anfibios son consideradas altamente variables, depauperadas y no interactivas (Aho, 1990; Barton, 1997; Luque et al., 2005; Koprivnikar et al., 2012). Son varios los factores que pueden influir sobre las poblaciones de metazoos parásitos en anfibios, tales como el ciclo biológico, dieta del hospedero, edad, tamaño y hábitat (Brooks et al., 2006; Hamann et al., 2006; Campião et al., 2014a,b). Los macropárasitos tienen un rico potencial como bioindicadores de modificaciones ambientales, especialmente en la cadena trófica (Koprivnikar et al., 2012).

En la región Neotropical se encuentra la mayor diversidad de anfibios (Frost, 1985; Izecksohn y Carvalho-Silva, 2001) y el Perú cuenta con alrededor de 561 especies descritas, situándose en tercer lugar, después de Brasil y Colombia, con respecto a riqueza de especies (AW, 2015). Sin embargo, los estudios referidos a conocer los metazoos pará- sitos de estos vertebrados en el Perú son muy escasos. La mayoría de ellos corresponde a descripciones taxonómicas de sus helmintos y pocos examinan las poblaciones y estructuras de sus comunidades (Bursey et al., 2001; Iannacone 2003a,b; Chero et al., 2014, 2015a,b).

La familia Bufonidae comprende alrededor de 49 géneros y 592 especies que se distribuyen alrededor del mundo, de las cuales cerca del 15\% (89 especies) pertenecen al género Rhinella, encontrándose 24 especies de este género en el Perú (AW, 2015). El «sapo espinoso» Rhinella spinulosa (Wiegmann, 1834) se distribuye en los Andes central y sur, en países como Argentina, Chile, Perú y Bolivia, con un amplio rango altitudinal desde el nivel del mar hasta los 5100 msnm (Angulo et al., 2010; Correa et al., 2010; Sanabria y Quiroga, 2011). Esta especie es muy usada en el centro del Perú como alimento y en medicina folclórica (Angulo et al., 2010). Según la lista roja de la Unión Internacional para la Conservación de la Naturaleza (UICN), $R$. spinulosa se encuentra 
en la categoría de preocupación menor (Angulo et al., 2010). Este anfibio se ha observado en muchas áreas naturales protegidas del Perú (Parque Nacional Huascarán, Santuario Nacional de Huayllay, Reserva Nacional de Junín, Santuario Histórico de Chacamarca y Aymara Lupaca, y Zona Reservada Alto Cañete Cochas Pachacayo) (Angulo et al., 2010).

Con relación a la fauna parasitaria del género Rhinella en Perú, se cuenta con información de helmintos parásitos en seis especies: $R$. arequipensis Vellard, 1959; $R$. limensis Werner, 1901; R. margaritifera Laurenti, 1768; $R$. marina Linnaeus, 1758; $R$. poeppigii Tschudi, 1945; y $R$. spinulosa. A la fecha, se han registrado 31 especies de parásitos en el Perú: un monogeneo [Wetapolystoma almae Gray, 1993], seis trematodos [Gorgoderina parvicava Travassos, 1922; Haematoloechus sp; Mosesia sp; Mesocoelium monas Rudolphi, 1819; Mesocoelium waltoni Brunetti, 1912; Phyllostomum (Microlecithus) sp], un cestodo [Cylindrotaenia americana Jewell, 1916], 23 nematodos [Aplectana hylambatis Baylis, 1927; Batracholandros spectatus Freitas \& Ibáñez 1962; Borrellostrongylus lumbrerasi Tantaleán \& Naupay, 1974; Cosmocerca brasiliense Travassos, 1925; Cosmocerca parva Travassos, 1925; Cosmocercoides dukae (Hall, 1928) Travassos, 1931; Falcaustra condorcanquii Ibáñez \& Córdova, 1976; Falcaustra mascula Rudolphi, 1819; Falcaustra sp, Hedruris moniezi Ibáñez \& Córdova, 1976; Hedruris sp; Ochoterenella digiticauda Caballero, 1944; Ochoterenella figueroai Esslinger, 1988; Oswaldocruzia manuensis Guerrero, 2013; Oswaldocruzia proencai Ben Slimane \& Durette Desset, 1995; Oswaldocruzia suburicularis Rudolphi, 1819; Oswaldocruzia urubambaensis Guerrero, 2013; Physaloptera huascari Ibáñez, 1973; Physaloptera sp; Raillietnema gubernaculum Freitas \& Ibáñez, 1965; Raillietnema ibañezi Cordova, 1998, Rhabdias aff. elegans Gutierrez, 1945; Rhabdias sphaerocephala Goodey, 1924] y un acantocefalo [Pseudoacanthocephalus lutzi (Hamann, 1891) Arredondo y Gil de Pertierra, 2009] (Ibáñez y Córdova, 1976; Ibáñez, 1998; Sarmiento et al., 1999; McAllister et al., 2010; Iannacone, 2003a,b; Guerrero, 2013; Amin y Heckmann, 2014; Fernandes y Kohn, 2014; Campião et al., 2014a; Chero et al., 2015a,b).

El objetivo de la presente investigación fue evaluar la comunidad de parásitos del sapo espinoso $R$. spinulosa en el Perú.

\section{Materiales y Métodos}

Se trabajó con 90 especímenes de $R$. spinulosa procedentes de Santa Eulalia, provincia de Huarochirí, Lima, Perú ( $11^{\circ} 542$

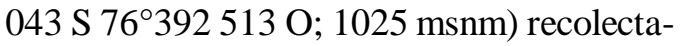
dos entre mayo de 2009 y octubre de 2010. Los especímenes fueron llevados al Laboratorio de Parasitología de la Universidad Nacional Federico Villarreal, Facultad de Ciencias Naturales y Matemática (UNFVFCCNM), Lima, Perú, en bolsas plásticas individuales, tratando que se mantuvieran en óptimo estado de conservación, para ser sacrificados mediante una solución de tiopental sódico. Fueron identificados siguiendo los criterios de Frost (1985) y de Garin y Hussein (2013). Los anfibios se identificaron como $R$. spinulosa (Bufonidae) por presentar una glándula parótida de tamaño mediano en el dorso, inmediatamente detrás de los ojos y piel muy áspera con formaciones glandulares cornificadas que le dan el aspecto de «espinas» (Urra, 2013).

La cavidad corporal, tubo digestivo (estómago, intestino delgado e intestino grueso), vejiga urinaria, pulmones y musculatura fueron examinados para la búsqueda de metazoos parásitos. Los helmintos colectados se fijaron, preservaron y montaron siguiendo las técnicas parasitológicas convencionales (Eiras et al., 2006). Los nemátodos fueron fijados en alcohol etílico al $70 \%$ caliente, los céstodos $\mathrm{y}$ trematodos se fijaron en formol al $10 \%$ bajo 
presión con dos láminas cubre-objetos, y los acantocéfalos se colocaron en agua destilada fría y se fijaron en formol al $10 \%$. Todos los parásitos fueron preservados en etanol $70 \%$.

Para la identificación taxonómica, los tremátodos, céstodos y acantocéfalos se colorearon con carmín acético de Semichon y se montaron en Entellan, mientras que los nematodos se clarificaron en una mezcla de alcohol etílico-fenol. Las especies fueron identificadas y clasificadas siguiendo literatura especializada (Ibañez y Córdova, 1976; Tantaléan y García, 1993; Anderson, 2000; Kuzmin et al., 2007; Arredondo y Gil de Pertierra, 2009; Pérez-Ponce de León et al., 2010; Santos et al., 2011; Amin, 2013; Tkach et al., 2013, 2014; Amin y Heckmann, 2014; Chero et al., 2014; Fernandes y Kohn, 2014). Material representativo de los helmintos parásitos colectados en el sapo espinoso se encuentra depositado en la Colección Científica de Protozoos y Metazoos Parásitos de la Universidad Nacional Federico Villarreal (CPMP-UNFV).

A cada hospedero se le determinó el sexo y la longitud total (LT) en centímetros. Las diferencias entre sexos de terminaron mediante la prueba estadística de «t» de Student, cumpliéndose previamente con la obligación de normalidad con la prueba de Kolmogorov-Smirnov con la modificación de Lillierfors y homocedasticidad de varianzas con la prueba de Levene. El 77\% $(n=69)$ de los hospederos fueron machos y el $23 \%$ $(\mathrm{n}=21)$ fueron hembras. Los especímenes presentaron una longitud total promedio de $11.01 \pm 1.27 \mathrm{~cm}$ (rango: $6.8-15.0 \mathrm{~cm}$ ), sin diferencias entre machos $(10.91 \pm 1.22 \mathrm{~cm}$, rango: $6.8-14.0 \mathrm{~cm})$ y hembras $(11.3 \pm 1.42$ $\mathrm{cm}$, rango: $10-15 \mathrm{~cm}$ ).

Los parámetros de infección parasitológicos (prevalencia [P], abundancia [AM] e intensidad media [IM] de infección) se analizaron según Bush et al. (1997). Se empleó el índice de importancia específica (IE) calculado como la importancia de cada especie parásita en el ensamblaje ecológico $[\mathrm{IE}=$ prevalencia + (abundancia media $\mathrm{x}$ 100)] con el fin de obtener un índice integrado de infección de ambos descriptores ecológicos (Bursey et al., 2001). La frecuencia de dominancia de cada especie parásita se determinó como el número de veces que es dominante una especie parásita en todos los hospederos examinados. La frecuencia de dominancia relativa de cada especie parásita fue considerada como el número de individuos de un taxón sobre el número total de individuos de todos los taxones en la infracomunidad parasitaria (Rohde et al., 1995).

Se emplearon tres índices de agregación con el fin de mostrar si los helmintos presentaban una distribución (1) contagiosa, agregada o conglomerada; (2) uniforme-regular o (3) aleatorizada (Iannacone et al., 2012). El análisis incluyó solo las especies de parásitos con prevalencias mayores al $10 \%$. Los índices fueron: (1) Dispersión (Id), (2) Índice de Discrepancia de Poulin ( $\left.\operatorname{ID}_{\mathrm{P}}\right)$, y (3) $\mathrm{K}$ de la ecuación binomial negativa (Bego y Von Zuben, 2010). Se aplicó el paquete Quantitative Parasitology 3.0 (Rózsa et al., 2000) para el cálculo de los tres índices de agregación.

El coeficiente de correlación de Spearman se usó para determinar la relación de la LT del hospedero con la AM de cada especie parásita. El coeficiente de correlación de Pearson se aplicó para evaluar la asociación entre la LT versus la $\mathrm{P}$ de infección, transformando previamente los valores de $\mathrm{P}$ a raíz cuadrada de arcoseno (Zar, 1996).

Se emplearon tablas de contingencia $2 \times 2$ para calcular el grado de asociación entre el sexo del hospedero y la $\mathrm{P}$ de cada parásito mediante la Prueba Exacta de Fisher. El análisis de los parásitos en relación con la talla y sexo del anfibio se realizó únicamente para las especies con prevalencias mayores al 10\% (Esch et al., 1990). Se usaron nueve índices de diversidad alfa: Riqueza, Simpson, Shannon, Brillouin, Menhinick, Margalef, 


\section{Pulmones:}

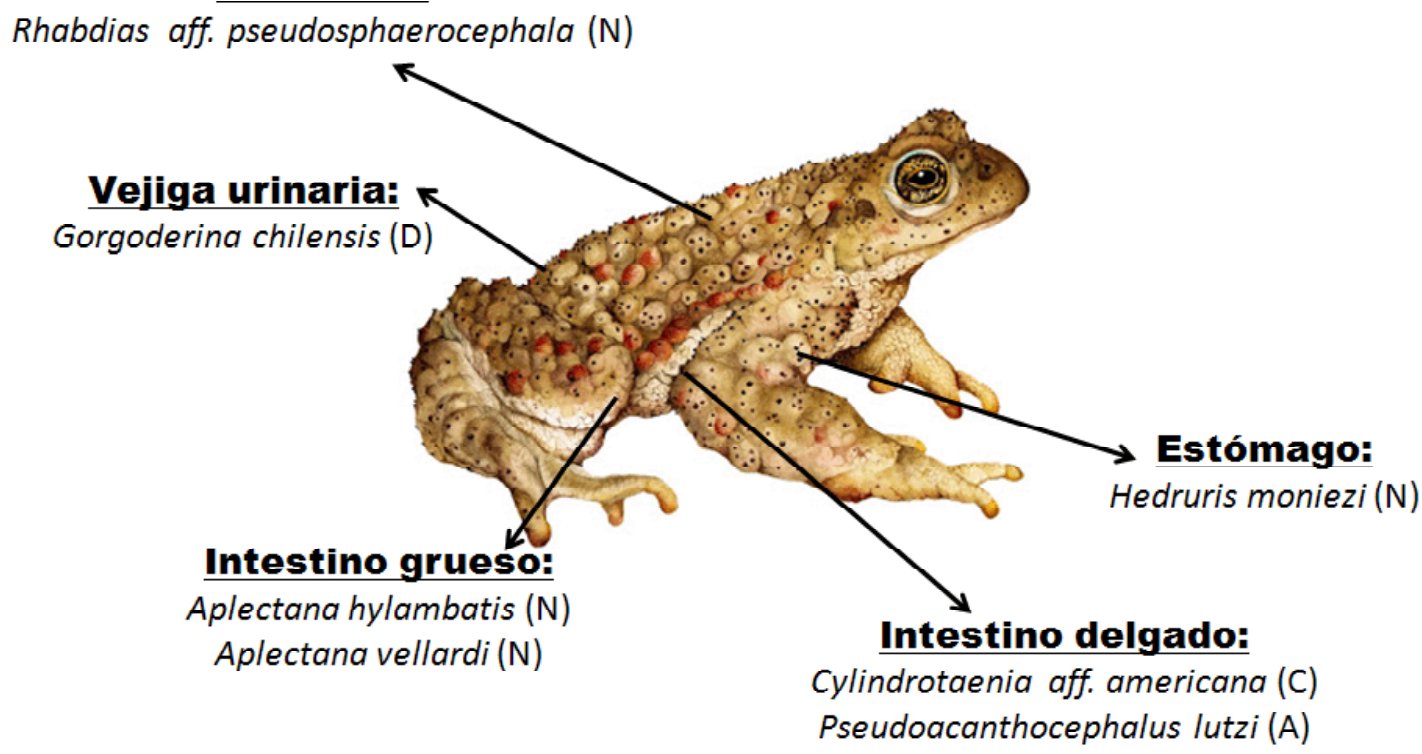

Figura 1. Espécimen de Rhinella spinulosa . Helmintos agrupados en hábitats. $\mathrm{N}=$ Nematoda. $\mathrm{D}=$ Digenea $. \mathrm{C}=$ Cestoda $. \mathrm{A}=$ Acantocephala (Fotografía: Andrea Ugarte)

Equitabilidad, Berger-Parker y Chao-1 (Moreno, 2001; Bego y Von Zuben, 2010) e individuos para el componente comunitario e infracomunidad parasitaria, tanto para el total como para sexo (Zar, 1996).

El índice de Chao-1 se empleó para estimar el número máximo de riqueza de helmintos parásitos a partir del esfuerzo de muestreo realizado y de la acumulación de nuevos taxa que van apareciendo en los hospederos muestreados (Moreno, 2001; Bego y Von Zuben, 2010). El dendrograma fue calculado con el índice de Ward en base a la distancia Euclidiana para comparar la similitud de los parásitos compartidos entre los 90 anfibios catastrados. Se empleó este índice, debido a que presentó el mayor valor del coeficiente de correlación. Para la determinación de los índices de diversidad, se usó el paquete estadístico PAST - Palaeontological Statistics v. 1.34 (Hammer et al., 2005). El nivel de significancia fue 0.05 . Para estos análisis se empleó el paquete estadístico IBM SPSS 21.0.

\section{Resultados}

Siete taxas de metazoos parásitos fueron indentificadas (Fig. 1): un digeneo [Gorgoderina chilensis Travassos, 1922 (Gorgoderidae)], un cestodo [Cylindrotaenia aff. americana Jewell, 1916 (Proteocephalidae)], cuatro nematodos [Aplectana hylambatis Baylis, 1927 (Cosmocercidae); A. vellardi (Cosmocercidae); Hedruris moniezi Ibáñez y Córdova, 1976 (Hedruridae) y Rhabdias aff. pseudosphaerocephala Kuzmin, Tkach y Brooks, 2007 (Rhabdiasidae)] y un acantocefalo [Pseudoacanthocephalus lutzi (Hamann, 1891) Arredondo y Gil de Pertierra, 2009 (Echinorhynchidae)]. 
Cuadro 1. Descriptores ecológicos, localización, estadio, ciclo de vida y código de depósito de siete parásitos del sapo espinoso Rhinella spinulosa, procedentes de Santa Eulalia, provincia de Huarochirí, Lima, Perú

\begin{tabular}{|c|c|c|c|c|c|c|c|}
\hline \multirow[b]{2}{*}{$\begin{array}{l}\text { Descrip- } \\
\text { tores }\end{array}$} & \multirow{2}{*}{$\begin{array}{l}\text { Trematoda } \\
\begin{array}{c}\text { Gorgoderina } \\
\text { chilensis }\end{array}\end{array}$} & \multirow{2}{*}{$\begin{array}{c}\text { Cestoda } \\
\text { Cylindro- } \\
\text { taenia aff. } \\
\text { americana }\end{array}$} & \multicolumn{4}{|c|}{ Nematoda } & \multirow{2}{*}{$\begin{array}{c}\text { Acantocephala } \\
\begin{array}{c}\text { Pseudoacantho- } \\
\text { cephalus lutzi }\end{array}\end{array}$} \\
\hline & & & $\begin{array}{l}\text { Rhabdias aff. } \\
\text { Pseudosphae- } \\
\text { rocephala }\end{array}$ & $\begin{array}{c}\text { Hedruris } \\
\text { moniezi }\end{array}$ & $\begin{array}{l}\text { Aplectana } \\
\text { hylambatis }\end{array}$ & $\begin{array}{c}\text { Aplectana } \\
\text { vellardi }\end{array}$ & \\
\hline $\begin{array}{l}\text { Ubicación } \\
\text { principal }\end{array}$ & $\begin{array}{c}\text { Vejiga } \\
\text { urinaria }\end{array}$ & $\begin{array}{c}\text { Intestino } \\
\text { delgado }\end{array}$ & Pulmones & Estómago & $\begin{array}{l}\text { Intestino } \\
\text { grueso }\end{array}$ & $\begin{array}{c}\text { Intestino } \\
\text { grueso }\end{array}$ & Inte stino delgado \\
\hline Estadio & Adulto & Adul to & Adulto & Adulto & Adulto & Adulto & Adulto \\
\hline $\begin{array}{l}\text { CPMP- } \\
\text { UNFV }\end{array}$ & $104-105$ & 106 & $107-108$ & 109 & 110 & 111 & $112-114$ \\
\hline $\mathrm{n}$ & 27 & 1 & 88 & 8 & 25 & 3 & 2 \\
\hline $\mathrm{N}$ & 83 & 8 & 705 & 47 & 670 & 45 & 56 \\
\hline $\mathrm{P}$ & 30 & 1.11 & 97.78 & 8.89 & 27.78 & 3.33 & 2.22 \\
\hline $\mathrm{AM}$ & 0.92 & 0.09 & 7.83 & 0.52 & 7.44 & 0.50 & 0.62 \\
\hline IM & 3.07 & 8 & 8.01 & 5.88 & 26.80 & 15 & 28 \\
\hline IE & 122 & 10.11 & 880.78 & 60.89 & 771.78 & 53.33 & 64.44 \\
\hline $\begin{array}{l}\text { Ciclo de } \\
\text { vida }\end{array}$ & Indirecto & Directo & Directo & Indirecto & $\begin{array}{l}\text { Directo/ } \\
\text { Indirecto? }\end{array}$ & $\begin{array}{l}\text { Directo/ } \\
\text { Indirecto? }\end{array}$ & Indirecto \\
\hline
\end{tabular}

$\mathrm{P}=$ Prevalencia. $\mathrm{IM}=$ Intensidad media de infección. $\mathrm{AM}=$ Abundancia media. $\mathrm{IE}=$ Índice de importancia específica. CPM P-UNFV = Colección de Protozoos y M etazoos Parásitos (CPM P) de la Universidad Nacional Federico Villarreal (UNFV). $\mathrm{n}=$ Número de hospederos infectados. $\mathrm{N}=$ Número total de parásitos

Los tres parásitos con mayor importancia específica (prevalencia y abundancia media) fueron los nematodos $R$. aff. pseudosphaerocephala $(\mathrm{n}=705 ; 44 \%)$ y $A$. hylambatis $(\mathrm{n}=670 ; 42 \%)$ y el trematodo digeneo G. chilensis ( $\mathrm{N}=83 ; 5 \%)$ (Cuadro 1). Los nematodos dominaron en número de especies $(n=4)$ en relación a los otros grupos taxonómicos. Todos los parásitos colectados correspondieron a formas adultas de helmintos.

La mayor frecuencia de dominancia y de dominancia relativa fueron para los nematodos $R$. aff. pseudosphaerocephala y A. hylambatis (Cuadro 2). Los tres parásitos más prevalentes ( $G$. chilensis, $R$. aff. pseudosphaerocephala y A. hylambatis) mostraron el típico patrón de distribución agregada observado en muchos sistemas hospedero-parásito (Cuadro 3). La prevalencia y abundancia media de todos los parásitos no estuvo significativamente correlacionada con la LT de los hospederos. Solo se observó una correlación positiva entre la LT de $R$. spinulosa, y la AM de $R$. aff. pseudosphaerocephala. La $\mathrm{P}$ de $G$. chilensis mostró dependencia con el sexo del anfibio; asimismo, se observó asociación significativa entre el sexo del anuro y la AM de G. chilensis, A. hylambatis y la AM del total de parásitos (Cuadro 3).

En el análisis parasitológico se recuperaron 1614 helmintos parásitos, con una prevalencia y abundancia media total del 100\% y 17.93, respectivamente. El promedio de la abundancia media total fue 17.93 (1-144). Asimismo, 52 hospederos (58\%) estuvieron parasitados por una especie, 14 (16\%) por dos especies, 23 (26\%) por tres especies y 1 (1\%) por cuatro especies. 
Cuadro 2. Frecuencia de dominancia y frecuencia de dominancia relativa de los parásitos componentes para el sapo espinoso Rhinella spinulosa, procedentes de Santa Eulalia, provincia de Huarochirí, Lima, Perú

\begin{tabular}{lccc}
\hline Parásito & $\begin{array}{c}\text { Frecuencia de } \\
\text { dominancia }\end{array}$ & $\begin{array}{c}\text { Frecuencia de } \\
\text { dominancia de dos } \\
\text { especies }\end{array}$ & $\begin{array}{c}\text { Frecuencia de } \\
\text { dominancia } \\
\text { relativa }\end{array}$ \\
\hline $\begin{array}{l}\text { Gorgoderina chilensis } \\
\text { Cylindrotaenia aff. americana }\end{array}$ & 4 & 14 & 0.05 \\
$\begin{array}{l}\text { Rhabdias aff. } \\
\text { pseudosphaerocephala }\end{array}$ & 68 & 1 & 0.00 \\
Hedruris moniezi & 2 & 30 & 0.44 \\
Aplectana hylambatis & 19 & 8 & 0.03 \\
Aplectana vellardi & 0 & 23 & 0.42 \\
Pseudoacanthocephalus lutzi & 1 & 2 & 0.03 \\
\hline
\end{tabular}

Cuadro 3. Coeficientes de correlación (r) usados para evaluar la relación entre la longitud total (LT) de Rhinella spinulosa vs la prevalencia (P) y la abundancia (A) de los endoparásitos. Prueba de $\mathrm{t}$ de student (t) y del estadístico de Fisher empleados para evaluar la relación entre el sexo de $R$. spinulosa y la abundancia y prevalencia de infección de endoparásitos. Valores de tres índices de agregación ( $\mathrm{Id}=$ Índice de Dispersión y $\mathrm{ID}_{\mathrm{p}}=$ Índice de Discrepancia de Poulin y K de la ecuación binomial negativa con su respectivo valor de $\mathrm{X}^{2}$ ) de los tres parásitos más prevalentes de $R$. spinulosa, procedentes de Santa Eulalia, provincia de Huarochirí, Lima, Perú

\begin{tabular}{|c|c|c|c|c|c|c|c|c|}
\hline \multirow{4}{*}{ Parásito } & $\mathrm{P}$ & $\mathrm{AM}$ & $\mathrm{P}$ & $\mathrm{AM}$ & \multirow{4}{*}{ Id } & \multirow{4}{*}{$\mathrm{IDp}$} & \multirow{4}{*}{$\mathrm{k}$} & \multirow{4}{*}{$\mathrm{X}^{2}$} \\
\hline & vs & vs & vs & vs & & & & \\
\hline & $\mathrm{LT}$ & LT & Sexo & Sexo & & & & \\
\hline & $r * / p$ & $r * * / p$ & Fisher(a) & $\mathrm{T}(\mathrm{b}) / \mathrm{p}$ & & & & \\
\hline Gorgoderina chilensis & $-0.50 / 0.66$ & $0.003 / 0.97$ & 0.02 & $2.75 / 0.007$ & 3.37 & 0.77 & 0.22 & 30.58 \\
\hline $\begin{array}{l}\text { Rhabdias aff. } \\
\text { pseudosphaerocephala }\end{array}$ & $0.00 / 1.00$ & $0.45 / 0.00$ & 1.00 & $2.42 / 0.01$ & 6.11 & 0.46 & 1.54 & 61.00 \\
\hline Aplectana hylambatis & $0.50 / 0.66$ & $0.006 / 0.96$ & 0.41 & $3.18 / 0.002$ & 61.56 & 0.88 & 0.07 & 40.68 \\
\hline Total de parásitos & & $0.14 / 0.17$ & & $4.69 / 0.000$ & 30.93 & 0.56 & 0.88 & 76.44 \\
\hline
\end{tabular}

$\mathrm{p}=$ nivel de significancia; $\mathrm{r}=$ coeficiente de correlación; $*$ = longitud total $v s$ prevalencia; $* *=$ longitud total $v s$ abundancia; (a) = comparar prevalencia de infección entre sexos; (b) = comparar la abundancia media entre sexos 
Cuadro 4. Índices de diversidad alfa parasitarios según componente comunitario e infracomunidades total y por sexo del sapo espinoso Rhinella spinulosa, procedentes de Santa Eulalia, provincia de Huarochirí, Lima, Perú

\begin{tabular}{|c|c|c|c|c|c|c|}
\hline \multirow[b]{2}{*}{ Índices } & \multicolumn{3}{|c|}{ Componente comunitario } & \multicolumn{3}{|c|}{ Infracomunidades } \\
\hline & $\begin{array}{c}\text { Total de } \\
\text { hospederos }\end{array}$ & Machos & Hembras & $\begin{array}{c}\text { Total de } \\
\text { hospederos }\end{array}$ & Machos & Hembras \\
\hline Riqueza & 7 & 7 & 3 & 1.65 & 1.76 & 1.28 \\
\hline Individuos & 1614 & 1490 & 124 & 17.93 & 21.59 & 5.90 \\
\hline Simpson & 0.42 & 0.44 & 0.32 & 0.81 & 0.79 & 0.90 \\
\hline Shannon H & 0.67 & 0.71 & 0.50 & 0.29 & 0.33 & 0.14 \\
\hline Brillouin & 0.59 & 0.59 & 0.39 & 0.24 & 0.27 & 0.11 \\
\hline Menhinick & 0.57 & 0.58 & 0.60 & 0.56 & 0.53 & 0.67 \\
\hline Margalef & 0.53 & 0.57 & 0.42 & 0.23 & 0.26 & 0.12 \\
\hline Equitabilidad $\mathrm{J}$ & 0.73 & 0.73 & 0.72 & 0.31 & 0.34 & 0.21 \\
\hline Berger-Parker & 0.68 & 0.66 & 0.80 & 0.86 & 0.83 & 0.93 \\
\hline Chao-1 & 8 & 8 & 3 & 2 & 2 & 1 \\
\hline $\begin{array}{l}\text { Especie más } \\
\text { abundante }\end{array}$ & $\begin{array}{l}\text { Rhabdias aff. } \\
\text { pse udospha- } \\
\text { erocephala }\end{array}$ & $\begin{array}{l}\text { Rhabdias aff. } \\
\text { pseudospha- } \\
\text { erocephala }\end{array}$ & $\begin{array}{l}\text { Rhabdias aff. } \\
\text { pseudospha- } \\
\text { erocephala }\end{array}$ & & & \\
\hline
\end{tabular}

El Cuadro 4 indica los valores de diversidad alfa según el total y el sexo en el componente comunitario y en las infracomunidades parasitarias de $R$. spinulosa. Se encontraron diferencias significativas en la mayoría de los índices, según el sexo del hospedero, siendo los índices mayores en machos que en hembras, a excepción de los índices de Simpson y Berger-Parker que presentaron valores ligeramente más altos en las hembras. En el caso del componente comunitario se notó valores intermedios de dominancia según Simpson y Berger-Parker, e índices altos de equitabilidad según Shannon, Brillouin y Equitabilidad.

Los valores de Chao-1 muestran que en el componente comunitario se requiere incrementar el tamaño de muestra, debido a que se espera encontrar hasta ocho especies $(13 \%+)$ y en el presente estudio solo se encontraron siete (Cuadro 4). Los valores de diversidad alfa de las infracomunidades parasitarias presentaron mayormente valores menores que el componente comunitario. En el dendrograma se observa una mayor asociación entre $H$. moniezi, $C$. aff. americana, G. chilensis, A. vellardi y $P$. lutzi, mientras que A. hylambatis y $R$. aff. pseudosphaerocephala presentaron menor asociación con el resto de los taxones (Fig. 2).

\section{Discusión}

En el presente trabajo se observó el predominio de los nematodos frente a los otros taxones de helmintos parásitos, al representar el $57 \%(n=4)$ del total de especies en el componente comunitario de $R$. spinulosa. Varios autores señalan la riqueza de especies de nematodos en la composición de las comunidades de parásitos de anfibios de la 


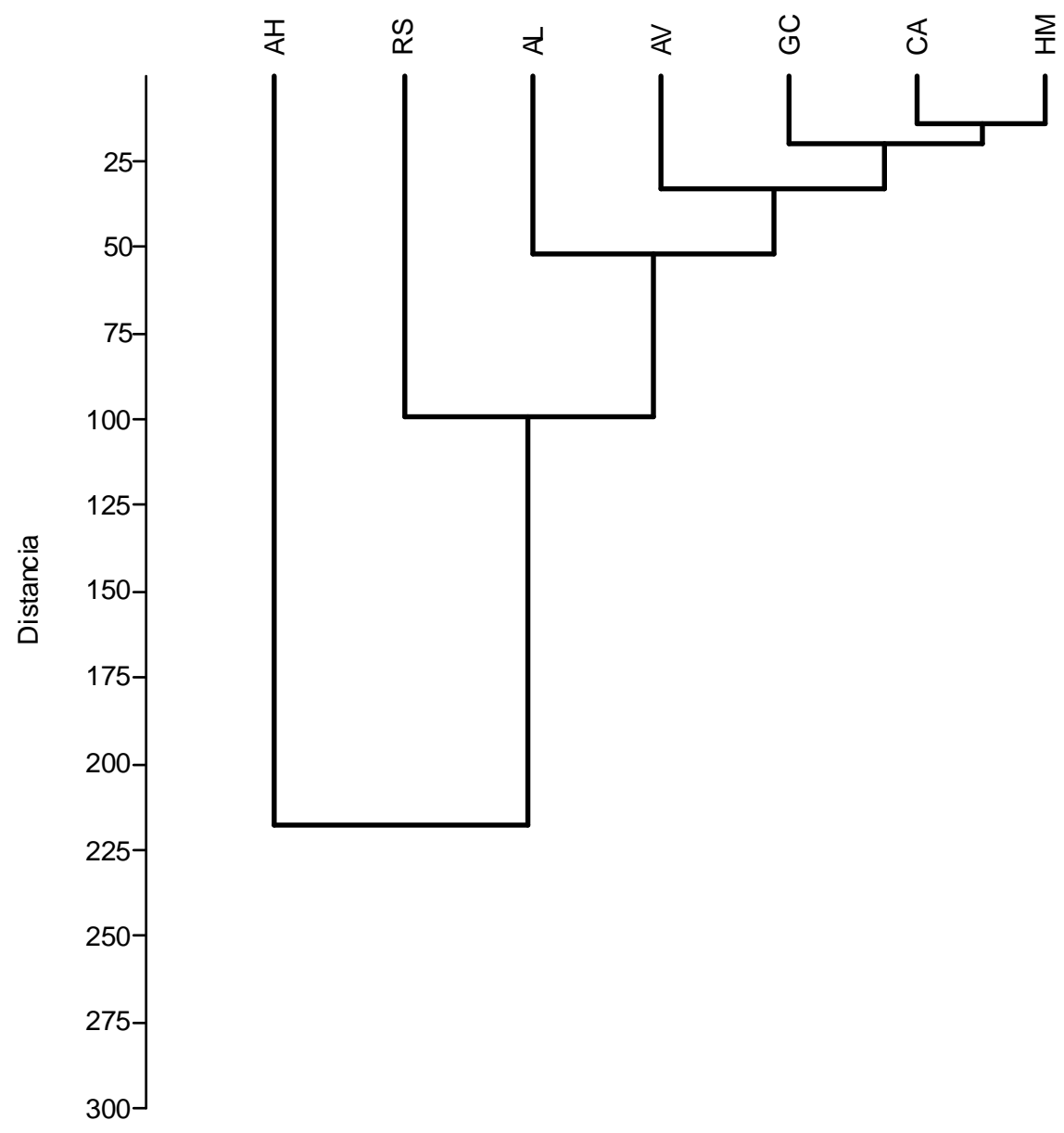

Figura 2. Dendrograma con el índice según el método de Ward mediante la distancia Euclidiana de asociación entre parásitos de Rhinella spinulosa $(\mathrm{r}=0.99)$, procedentes de Santa Eulalia, provincia de Huarochirí, Lima, Perú. GC = Gorgoderina chilensis; $\mathrm{CA}=$ Cylindrotaenia aff. americana; $\mathrm{RP}=$ Rhabdias aff. pseudosphaerocephala; $\mathrm{HM}$ $=$ Hedruris moniezi $; \mathrm{AH}=$ Aplectana hylambatis $; \mathrm{AV}=$ Aplectana vellardi $; \mathrm{PL}=$ Pseudoacantocephalus lutzi

familia Bufonidae (Iannacone, 2003a; Ragoo y Omah-Maharaj, 2003; Luque et al., 2005; Pinhao et al., 2009; Santos y Amato, 2010). Además, los anuros terrestres presentan mayor frecuencia de infección por un gran número de nematodos, en contraste con los anuros acuáticos que albergan gran cantidad de digeneos (metacercarias y adultos) (Barton, 1997; Iannacone, 2003a; Santos y Amato, 2010; Chero et al., 2014, 2015a,b), debido a que los primeros se alimentan principalmente de himenópteros, coleópteros y otros invertebrados terrestres, que estarían actuando como hospederos intermediarios de diferentes especies de nematodos con ciclos heteroxénicos (Luque et al., 2005). Esto podría explicar la razón por la que el bufonido $R$. spinulosa, que es una especie mayormente terrestre, estaría albergando cuatro especies de nematodos de un total de siete especies registradas.

En $R$. icterica (Spix, 1824) de Rio de Janeiro (Brasil), se ha reportado la dominancia de nematodos con trece especies que representan el $81 \%$ del total de especies re- 
portadas (Luque et al., 2005). Iannacone (2003b), al revisar los helmintos parásitos de Atelopus aff. bomolochus del departamento de Piura (Perú), muestra que la comunidad de helmintos está dominada por cuatro especies de nematodos. En el bufonido argentino $R$. schneideri Werner, 1894 se encontraron hasta ocho especies de nematodos que estarían estructurando su componente comunitario (González y Hamann, 2008).

Los trematodos del género Gorgoderina parasitan la vejiga urinaria de anuros y caudados (Amphibia) a nivel mundial (MataLópez et al., 2005; Lunaschi y Drago, 2010). G. chilensis se le ha encontrado parasitando a nivel intestinal y vejiga urinaria de Rhinoderma darwini (Duméril y Bibron, 1841) (Rhinodermaridae) en Chile y Uruguay (Mata-López et al., 2005; Olmos y Muñoz, 2006; Campião et al., 2014a) y Telmatobius brachydactylus (Peters, 1873) en Perú (Tantaléan y García, 1993; Fernández y Kohn, 2014). En el presente estudio, la $\mathrm{P}$ y la AM de G. chilensis se encontró en asociación con el sexo de $R$. spinulosa, siendo las hembras las más infectadas. Opuestamente, Saeed et al. (2007) encontraron que la prevalencia de Gorgoderina sp fue mayor en anfibios machos que en hembras, atribuyendo tal variación a factores intrínsecos que podrían tener un impacto en el comportamiento de los organismos de ambos sexos en respuesta a los factores ecológicos. G. chilensis es el segundo registro para el Perú parasitando a $R$. spinulosa.

Los nematodos del género Rhabdias son parásitos pulmonares con un ciclo biológico directo complejo (infección de anfibio a anfibio) (Kuzmin et al., 2007; Saeed et al., 2007; Saad et al., 2009; Santos et al., 2011), que parasitan principalmente ránidos y bufónidos (Mohammad et al., 2010). Sin embargo, también se les encuentra parasitando salamandras, serpientes y lagartijas en regiones tropicales y templadas (González y Hamann, 2008; Santos et al., 2011). Campião et al. (2014a) señalan que se han registrado ocho especies de Rhabdias en Sudamérica
(R. androgyna Kloss, 1971; R. elegans Gutiérrez, 1945; R. fuelleborni Travassos, 1926; $R$. hermafrodita Kloss, 1971; $R$. mucronata Schuurmans Stekhoven, 1952; $R$. paraensis Santos, Melo, Nascimento, Nascimento, Giese \& Furtado, 2011; $R$. sphaerocephala [Goodey, 1924] y $R$. truncata Schuurmans Stekhoven, 1952). En el Perú, solo se ha registrado a $R$. sphaerocephala en Atelopus aff. bomolochus y $R$. spinulosa, a $R$. aff. elegans Kloss, 1971 en Rhinella poeppigii (Tschudi, 1845) y a $R$. fuelleborni en Rhinella limensis (Werner, 1901) (Campião et al., 2014a; Chero et al., 2015a,b).

La identificación taxonómica de Rhabdias de anfibios es complicada, debido a la uniformidad morfológica de las especies y ausencia de machos en la generación parasítica. Martínez-Salazar et al. (2009) señalan que la morfología conservativa de Rhabdias ha resultado en una incorrecta identificación de algunas especies, por lo que se requiere incorporar nuevas herramientas taxonómicas (marcadores moleculares y microscopía electrónica de barrido), así como considerar la especificidad al hospedero y distribución geográfica, debido a que raramente son parásitos de más de un grupo hospedero. El principal carácter para la diferenciación de las especies de Rhabdias de anfibios es la longitud relativa del esófago en relación a su longitud total (Santos et al., 2011; Tkach et al., 2014). Debido a este carácter diferencial, los especímenes del presente estudio no corresponden a la especie europea $R$. sphaerocephala, sino a $R$. aff. pseudosphaerocephala Kuzmin, Tkach y Brooks, 2007. Esta última no ha sido registrada antes en el Perú, sino en Centroamérica (Nicaragua y Costa Rica), México, y Brasil en los pulmones de $R$. marina (Linneaus, 1758). Sin embargo, faltaría aclarar si todos los registros de $R$. sphaerocephala en $R$. spinulosa (sin. Bufo flavolineatus y $B$. trifolium) indicados por Sarmiento et al. (1999), para seis departamentos del Perú (Arequipa, Cusco, Ica, Huánuco, Junín y Pasco), corresponden también a $R$. aff. 
pseudosphaerocephala o a una especie de Rhabdias aún no descrita (Campiao et al., 2014a). Rhabdias aff. pseudosphaerocephala es un nuevo registro para $R$. spinulosa en el Perú y el segundo para Sudamérica. Lettoof et al. (2013) encontraron que la prevalencia de $R$. pseudosphaerocephala en $R$. marina fue de $70.5 \%$, mayor a la observada en varios anuros nativos de Australia (0-38.5\%).

Se observó una correlación positiva entre la talla y sexo con la abundancia media de $R$. aff. pseudosphaerocephala. Luque et al. (2005) señalaron que un aumento en la $\mathrm{P}$ y AM podría ser esperado en los hospederos anuros de mayor tamaño que presentan una mayor superficie e ingieren una dieta más diversa. Pizzatto et al. (2013) indicaron que $R$. pseudosphaerocephala en $R$. marina en Australia incrementa su P e IM con el tamaño corporal del hospedero, pero presentó ausencia de relación con el sexo.

Hedruris moniezi ha sido descrito para el Perú para tres especies de anfibios del género Telmatobius ( $T$. jelksii, $T$. marmoratus y $T$. peruvianus), con una prevalencia de 78.6-90.0\% (Chero et al., 2014), en tanto que en el presente estudio se encontró una prevalencia de $8.89 \%$ en $R$. spinulosa. El género Hedruris presenta como hospederos intermediarios a crustáceos isópodos y anfípodos a nivel del hemocele (Luque et al., 2010; Rauque, 2013). Sarmiento et al. (1999) registraron previamente a $H$. moniezi en $R$. spinulosa.

El nematodo cosmocercido, $A$. hylambatis, presenta una amplia distribución y ha sido reportado en África y América del Sur, en países como Argentina, Ecuador, Guyana, Paraguay, Perú y Uruguay (Campião et al., 2014a), parasitando el intestino de anuros (Iannacone 2003a,b; Chero et $a l .$, 2014). En el Perú se le registrado en 11 especies y cinco familias de anfibios: Atelopus aff. bomolochus, $R$. arequipensis Vellard, 1959, R. marina, R. poeppigii, R. spinulosa (Bufonidae), Gastrotheca marsupiata
(Duméril \& Bibron, 1841) (Hemiphractidae), Leptodactylus rhodonotus Günther, 1868 (Leptodactylidae), Pleurodema marmorata (Duméril \& Bibron, 1841) (Leiuperidae), $T$. jelskii, T. peruvianus y T. marmoratus (Telmatobiidae) (Sarmiento et al., 1999; Iannacone, 2003a,b; Campião et al., 2014a; Chero et al., 2014, 2015b).

El género Aplectana presenta un ciclo de vida directo. En el intestino del anfibio hospedero se localizan las hembras que producen larvas, las cuales son expulsadas junto con las heces del anuro y depositadas en el agua, donde se desarrollan las larvas $\mathrm{L}_{2} \mathrm{y} \mathrm{L}_{3}$. Esta última penetra a través de la piel del hospedero y migra hacia el recto. Las larvas que se encuentran en el ambiente acuático también pueden ser ingeridas por renacuajos y desarrollar el adulto. Además, los anuros adultos pueden infectarse también, si es que estos últimos ingieren a los renacuajos portadores de este nematodo (Anderson, 2000; Pérez-Ponce de León et al., 2010). Sin embargo, Rodríguez-Amador et al. (2013) señalan que la infección por Aplectana sp en el anfibio Ambystoma velasci Dugès, 1888, está relacionada con la presencia de insectos, lo que sugiere un ciclo biológico indirecto.

En el Perú, se han registrado prevalencias de 6.1 y $3.0 \%$ de $A$. hylambatis en $A$. aff. bomolochus y $T$. jelskii, respectivamente (Iannacone, 2003a,b); sin embargo, los resultados para el bufónido $R$. spinulosa indicaron una prevalencia de $27.78 \%$. En una especie congenérica, $R$. arenarum Hensel, 1867, se ha señalado a $A$. hylambatis como una especie dominante con una prevalencia del 62.5\% (González et al., 2013). Para otras especies del género Aplectana que parasitan a rinélidos sudamericanos, también se han registrado altas prevalencias de infección (Luque et al., 2005; Madelaire et al., 2012). De igual forma, se observó asociación entre el sexo del anuro y la abundancia media de A. hylambatis, presentándose valores mayores en los machos que en las hembras. Chero et al. (2014) indican que el aumento de los 
niveles de testosterona en los anfibios machos puede influenciar positivamente la carga de helmintos parásitos en los anfibios.

Pseudoacanthocephalus lutzi fue descrita inicialmente como Echinorhynchus lutzi por Hamann (1891) en el intestino del bufónido R. marina (=Bufo agua) de Brasil. Posteriormente, Meyer (1932) transfiere a $E$. lutzi al género Acanthocephalus (Amin y Heckmann, 2014). Bursey y Goldberg (2007) reasignan a A. lutzi como Anuracanthorhynchus lutzi basados en especímenes colectados de Lithobates warszewitschii Schmidt, 1857, de Costa Rica, tomando como caracteres taxonómicos el número de glándulas de cemento, la forma de los testículos, la ausencia de espinas en el tronco y ganchos de la probóscide de igual longitud. Sin embargo, Arredondo y Gil de Pertierra (2009) rechazan esta propuesta y reasignan a $A$. lutzi al género Pseudoacanthocephalus basados en el material tipo de $R$. marina de Brasil y material colectado de $R$. arenarum Hensel, 1867, de Argentina. Además, señalan los caracteres diagnósticos del género, que incluyen la presencia de un tronco cilíndrico sin espinas, una probóscide cilíndrica con armadura característica, testículos en «tándem», mayormente seis glándulas de cemento compactas, poro genital masculino casi terminal, poro genital femenino ventral y sub-terminal, y huevos sin prolongaciones polares, que contienen un acantor tipo holoechineto.

El género Pseudoacanthocephalus actualmente tiene 18 especies conocidas, siendo dos sudamericanas: $P$. lutzi y $P$. caspanensis (Fernández e Ibarra Vidal, 1992) (Amin et al., 2014; Tkach et al., 2014). $P$. lutzi ha sido registrada en cuatro especies de anfibios bufónidos en el Perú ( $R$. spinulosa, $R$. marina, $R$. arequipensis y $R$. limensis) (Tantaleán et al., 2005). Arredondo y Gil de Pertierra (2009) señalan que es necesario reevaluar el material peruano correspondiente con P. lutzi. Por ende, Amin y Heckmann (2014) describen el material pe- ruano de $P$. lutzi de $R$. limensis (Werner, 1901) y proporcionan nuevos detalles mediante microscopía electrónica de barrido de su morfología externa y estructuras internas. Este helminto con ciclo indirecto parece tener un ciclo de vida semiacuático, debido a que sus huevos tienen un cubierta fibrilar y por carecer de una cobertura E3d típica de archiacantocéfalos y porque la dieta del hospedero incluye insectos acuáticos, como los Odonata (Arredondo y Gil de Pertierra, 2009; Tkach et al., 2013).

Los tres parásitos más prevalentes de $R$. spinulosa indicaron para todos los casos un patrón de distribución contagioso. Chero et al. (2014) señalan que este comportamiento de distribución contagiosa se ha observado en las comunidades helmínticas en varios anuros de Sudamérica. Los valores de diversidad alfa de las infracomunidades parasitarias presentaron mayormente valores menores que el componente comunitario. Campião et al. (2014b) encontraron un patrón opuesto con valores del componente comunitario más bajos que la infracomunidad parasitaria, a excepción de la riqueza de especies para las comunidades helmínticas de Leptodactylus podicipinus (Cope, 1862) en Brasil.

Se observó una mayor asociación entre H. moniezi, C. aff. americana, G. chilensis, $A$. vellardi y $P$. lutzi, mientras que $A$. hylambatis y $R$. aff. pseudosphaerocephala presentaron menor asociación con el resto de los taxones. Las cinco especies que presentaron mayor asociación fueron las que presentaron la menor prevalencia. Tres de estas especies: H. moniezi, G. chilensis y $P$. lutzi presentaron ciclo biológico indirecto. En cambio, las dos especies con menor asociación, A. hylambatis y $R$. aff. pseudosphaerocephala, presentaron ciclo biológico directo y mayores prevalencias y frecuencias de dominancia. Chero et al. (2014) encontraron en $T$. jelskii y $T$. marmoratus una mayor asociación entre $H$. moniezi y A. hylambatis. 


\section{Literatura Citada}

1. Aho JM. 1990. Helminth communities of amphibians and reptiles: comparative approaches to understanding patterns and processes. In: Esch GW, Bush AO, Aho JM (eds). Parasite communities: patterns and processes. New York, USA: Chapman and Hall. p 157-190.

2. Anderson $\boldsymbol{R}$ C. 2000. Nematode parasites of vertebrates. Their development and transmission. $2^{\text {nd }} e d$. Wallingford, UK: CABI Publishing. $672 \mathrm{p}$.

3. Angulo A, De la Riva I, Córdova-Santa Gadea J, Veloso A, Núñez, H, Úbeda C, et al. 2010. Rhinella spinulosa. The IUCN Red List of Threatened Species. [Internet]. Disponible en: http:// www.iucnredlist.org/details/54763/0. doi: 10.2305/IUCN.UK.2010-2.RLTS. T54763A11186587.en

4. Amin OM. 2013. Classification of the Acanthocephala. Folia Parasitol 60: 273305. doi: 10.14411/fp.2013.031

5. Amin OM, Heckmann RA. 2014. First description of Pseudoacanthocephalus lutzi from Peru using SEM. Sci Parasitol 15: $19-26$.

6. Amin OM, Heckmann RA, Van Ha N. 2014. Acantocephalan from fishes and amphibians in Vietnam, with descriptions of five new species. Parasite 21: 53. doi: 10.1051/parasite/2014052

7. Arredondo NJ, Giel de Pertierra AA. 2009. Pseudoacanthocephalus lutzi (Hamann, 1891) comb. n. (Acanthocephala:Echinorhynchidae)for Acanthocephalus lutzi (Hamann, 1891), parasite of South American amphibians. Folia Parasitol 56: 295-304.

8. AW [AmphibiaWeb]. 2015. Information on amphibian biology and conservation. [Internet]. Available in : http:// amphibiaweb.org/

9. Barton DP. 1997. Why are amphibian helminth communities depauperate? Mem Mus Vic 56: 581-586.
10. Bego NM, Von Zuben CJ. 2010. Métodos quantitativos em parasitologia. Jaboticabal, Brazil: FUNEP. 72 p.

11. Brooks DR, Leon-Regagnon V, Mc Lennan DA, Zelmer D. 2006. Ecological fitting as a determinant of the community structure of platyhelminth parasites of anurans. Ecology 87: S76S85.

12. Bursey CR, Goldberg SR, Parmelee, JR. 2001. Gastrointestinal helminths of 51 species of anurans from Reserva Cuzco Amazónico, Peru. Comp Parasitol 68:21-35.

13. Bursey CR, Goldberg SR. 2007. New species of Hedruris (Nematoda: Hedruridae), Anuracanthorhynchus lutzi (Hamann, 1891) n. comb. and other Helminths in Lithobates warszewitschii (Anura: Ranidae) from Costa Rica. Caribb J Sci 43: 1-10.

14. Bush AO, Lafferty KD, Lotz JL, Shostak AW. 1997. Parasitology meets ecology on its own terms: Margolis et al. revisited. J Parasitol 83: 575-583. doi: $10.2307 / 3284227$

15. Campião KM, Morais DH, Dias OT, Aguiar A, Toledo GDM, Tavares LER, Da Silva RJ. 2014a. Checklist of helminth parasites of amphibians from South America. Zootaxa 3843: 1-93. doi: 10.11646/zootaxa.3843.1.1

16. Campião KM, Da Silva RJ, Ferreira $V \boldsymbol{L}$. 2014b. Helminth parasite communities of allopatric populations of the frog Leptodactylus podicipinus from Pantanal, Brazil. J Helminthol 88: 13-19. doi: 10.1017/S0022149X12000557

17. Chero J, Cruces C, Iannacone J, Sáez G Alvariño L, da Silva RJ, Morales VR. 2014. Gastrointestinal parasites in three species of Telmatobius (Anura: Telmatobiidae) of an area of high Andes, Peru. Neotrop Helminthol 8: 439-461.

18. Chero J, Cruces C, Iannacone J, Sáez G, Alvariño L, Silva RJ, Morales V, Minaya D. 2015a. Parasitofauna of the neotropical amphibian Rhinella limensis Werner, 1901 (Anura: Bufonidae) from 
Peruvian central coast. Neotrop Helminthol 9: 87-102.

19. Chero J, Cruces C, Iannacone J, Sáez $G$ Alvariño l, Guabloche A, Romero $S$, et al. 2015b. Índices ecológicos de los helmintos parásitos del sapo andino Rhinella poeppigii (Tschudi, 1845) (Anura: Bufonidae) del Perú. Biologist (Lima) 13: 111-124.

20. Correa C, Pastenes L, Sallaberry M, Veloso A, Méndez MA. 2010. Phylogeography of Rhinella spinulosa (Anura: Bufonidae) in northern Chile. Amphib-Reptil 31: 85-96.

21. Eiras JC, Takemoto, RM, Pavanelli GC. 2006. Métodos de estudo e técnicas laboratoriais em parasitologia de peixes. Maringá: Eduem. 305 p.

22. Esch GW, Shostak AW, Marcogliese DJ, Goater TM. 1990. Patterns and process in helminth parasite communities: an overview. In: Esch G, Bush AC, Aho J (eds). Parasite communities: patterns and processes. New York, USA: Chapman and Hall. p 1-19.

23. Fernandes BMM, Kohn A. 2014. South American trematodes parasites of amphibians and reptiles. Rio de Janeiro: Instituto Oswaldo Cruz. 228 p.

24. Frost DR. 1985. Amphibian species of the world, a taxonomic and geographical reference. Kansas, USA: Allen Press. $732 \mathrm{p}$.

25. Garin CF, Hussein Y. 2013. Guía de reconocimiento de anfibios y reptiles de la región de Valparaíso. Chile: Servicio Agrícola y Ganadero (SAG). 63 p.

26. González CE, Hamann MI. 2008. Nematode parasites of two anuran species Rhinella schneideri (Bufonidae) and Scinax acuminatus (Hylidae) from Corrientes, Argentina. Rev Biol Trop 56: 2147-2161. doi: 10.15517/ rbt.v56i4.5785

27. González CE, Quiroga LB, Moreno D, Sanabria EA. 2013. Primer registro de Aplectana hylambatis (Nematoda, Cosmocercidae) para anfibios de la provincia de San Juan. Cuad Herpetol 27: 155-159.
28. Guerrero R. 2013. Two new species of Oswaldocruzia (Nematoda: Trichostrongylina: Molineoidea) parasites of the cane toad Rhinella marina (Amphibia: Anura) from Peru. Acta Parasitol 58: 3036. doi: 10.2478/s11686-013-0103-4

29. Hamann M, González C, Kehr A. 2006. Helminth community structure of the oven frog Leptodactylus latinasus (Anura, Leptodactylidae) from Corrientes, Argentina. Acta Parasitol 51: 294299. doi: 10.2478/s11686-006-0045-1

30. Hammer $\emptyset$, Harper DAT, Ryan PD. 2005. PAST: Paleontological Statistics Software Package for Education and Data Analysis. Palaeo Electrónica 4: 9 [Internet]. Available in: http://palaeoelectronica.org/2001_1/past/past.pdf

31. Ibáñez HN, Córdova BE. 1976. Cuatro especies nuevas de nematodos del sur del Perú y redescripción de Hedruris orestiae Moniez, 1889. Mem Inst Oswaldo Cruz 74: 231-253. doi: 10.1590/ S0074-02761976000300004

32. Ibáñez NH. 1998. Mención de algunos trematodos en la fauna helmintológica peruana. Rev Per Parasitol 13: 90-97.

33. Iannacone J. 2003a. Helmintos parásitos de Telmatobius jelskii (Peters) (Anura, Leptodactylidae) de Lima, Perú. Rev Bras Zool 20: 131-134. doi: 10.1590/ S0101-81752003000100016

34. Iannacone J. 2003b. Helmintos parasitos de Atelopus bomolochus Peters 1973 (Anura: Bufonidae) de Piura, Peru. Gayana 67: 9-15. doi: 10.4067/ S0717-65382003000100002

35. Iannacone J, Sánchez V, Olazábal N, Salvador C, Alvariño L, Molano J. 2012. Ecological indices of parasites of Scartichthys gigas (Steindachner, 1876) (Perciformes: Blenniidae) of the coasts of Lima, Peru. Neotrop Helminthol 6: 191-203.

36. Izechsohn E, Carvalho e Silva SP. 2001. Anfíbios do Município do Rio de Janeiro. Rio de Janeiro, RJ: Ed UFRJ. $147 \mathrm{p}$. 
37. Koprivnikar J, Marcogliese DJ, Rohr JR, Orlofske SA, Raffel TR, Johnson PTJ. 2012. Macroparasite infections of amphibians: what can they tell us? Ecohealth 9: 342-360. doi: 10.1007/ s10393-012-0785-3

38. Kuzmin Y, Tkach VV, Brooks DR. 2007. Two new species of Rhabdias (Nematoda: Rhabdiasidae) from the marine toad, Bufo marinus (L.) (Lissamphibia: Anura: Bufonidae), in Central America. J Parasitol 93: 159-165.

39. Lettoof DA, Greenlees MJ, Stockwell M, Shine R. 2013. Do invasive cane toads affect the parasite burdens of native Australian frogs? Int J Parasitol Parasites Wildl 2: 155-164. doi: 10.1016/ j.ijppaw.2013.04.002

40. Luque JL, Martins AN, Tavares ER. 2005. Community structure of metazoan parasites of the yellow cururu toad, Bufo ictericus (Anura, Bufonidae) from Rio de Janeiro, Brazil. Acta Parasitol 50: 215220.

41. Luque JL, Vieira FM, Herrmann K, King TM, Poulin R, Lagrue C. 2010. New evidence on a cold case: trophic transmission, distribution and hostspecificity in Hedruris spinigera (Nematoda: Hedruridae). Folia Parasitol (Praha) 57: 223-231.

42. Lunaschi LI, Drago FB. 2010. Platyhelminthes, Trematoda, Digenea Carus, 1863: Distribution extension in Argentina and new Anura and Ophidia hosts. Check List 6: 447-450.

43. Madelaire CB, Gomes FR, da Silva RJ. 2012. Helminth parasites of Hypsiboas prasinus (Anura: Hylidae) from two Atlantic forest fragments, São Paulo State, Brazil. J Parasitol 98: 560564. doi: 10.1645/JP-GE-2665.1

44. Martínez-Salazar EA, Pérez-Ponce de León G, Olea GP. 2009. First record of the genus Rhabdias (Nematoda: Rhabdiasidae), endoparasite from Scinax staufferi (Anura: Hylidae) in Mexico. Rev Mex Biodivers 80: 861-865.
45. Mata-López R, León-Règagnon V, Brooks D. 2005. Species of Gorgoderina (Digenea: Gorgoderidae) in Rana vaillanti and Rana cf. forreri (Anura: Ranidae) from Guanacaste, Costa Rica, including a description of a new species. J Parasitol 91: 403-410.

46. McAllister CT, Bursey CR, Freed PS. 2010. Helminth parasites of amphibians and reptiles from the Ucayali region, Peru. J Parasitol 96: 444-447. doi: 10.1645/GE-2206.1

47. Mohammad MK, Al-Moussawi AA, Jasim SY. 2010. Helminth parasites of the green toad Bufo viridis Laurenti, 1768 in Baghdad Area, Central Iraq. Egypt Acad J Biolog Sci 2(1): 17-25.

48. Moreno E. 2001. Métodos para medir la biodiversidad. Zaragoza, España: M\&T - Manuales y Tesis SEA. $84 \mathrm{p}$.

49. Olmos V, Muñoz G. 2006. Estado de conocimiento de los parásitos eumetazoos de organismos dulceacuícolas de Chile. Gayana 70: 122-139. doi: 10.4067/ S0717-653820060-00100018

50. Pérez-Ponce de León G, García-Prieto L, Mendoza-Garfias B. 2010. Helmintos parásitos de vertebrados silvestres. En: Molina-Freaner FE, van Devender TR (eds.). Diversidad biológica de Sonora. México DF: Universidad Nacional Autónoma de México (UNAM). p 263-283.

51. Pinhao $R$, Wunderlich AC, dos Anjos LA, da Silva RJ. 2009. Helminths of toad Rhinella icterica (Bufonidae), from the Municipality of Botucatu, Sao Paulo state, Brazil. Neotropical Helminthol 3: $35-40$.

52. Pizzatto L, Kelehear C, Shine R. 2013. Seasonal dynamics of the lungworm, Rhabdias pseudosphaerocephala, in recently colonized cane toad (Rhinella marina) populations in tropical Australia. Int J Parasitol 43: 753-761. doi: 10.1016/j.ijpara.2013.05.002

53. Ragoo RM, Omah-Maharaj IR. 2003. Helminths of the cane toad Bufo marinus from Trinidad, West Indies. Caribb J Sci 39: 242-245. 
54. Rauque CA. 2013. Rol de los anfípodos (Amphipoda) en ambientes de agua dulce de Patagonia: epibiosis y parasitismo. Rev Arg Parasitol 2: 6-15.

55. Rodhe K, Hayward C, Heap M. 1995. Aspects of the ecology of metazoan ectoparasites of marine fishes. Int $\mathbf{J}$ Parasitol 25: 945-970.

56. Rodríguez-Amador R, Monks S, Pulido-Flores G. 2013. Primer reporte helmintológico de Ambystoma velasci Dugés, 1888 de Lago de Tecocomulco, Hidalgo, México. En: Pulido-Flores G, Monks S (eds.). Estudios científicos en el estado de Hidalgo y zonas aledañas. Vol II. Lincoln, Nebraska: Zea E-Books. p 113-120.

57. Rózsa L, Reiczigel J, Majoros G. 2000. Quantifying parasites in samples of hosts. J Parasitol 86: 228-232.

58. Saad AI, Khalifa R, Mostafa N. 2009. A lungworm nematode from amphibian hosts at Aswam Governorate, Egypt. World J Zool 4: 37-41.

59. Saeed I, Al-Barwari SE, Al-Harmni KI. 2007. A metazoan parasitological research of some Iraqi amphibians. Turkiye Parazitol Derg 31: 337-345.

60. Sanabria EA, Quiroga LB. 2011. Facultative nesting in Rhinella spinulosa (Anura: Bufonidae): strategy to avoid dehydration of offspring. Belg $\mathrm{J}$ Zool 141: 85-89.

61. Santos JN, Melo FTV, Nascimento LCS, Nascimento, DEB, Giese EG, Furtado AP. 2011. Rhabdias paraensis sp. now.: a parasite of the lungs of Rhinella marina (Amphibia: Bufonidae) from Brazilian Amazonia. Mem Inst Oswaldo Cruz 106: 433-440. doi: 10.1590/S0074-02762011000400008
62. Santos VG, Amato SB. 2010. Helminth fauna of Rhinella fernandezae (Anura: Bufonidae) from the Rio Grande do Sul coastland, Brazil: analysis of the parasite community. J Parasitol 96: 823-826. doi: 10.1645/GE-2388.1

63. Sarmiento L, Tantaleán M, Huiza A. 1999. Nemátodos parásitos del hombre y de los animales en el Perú. Rev Per Parasitol 14: 9-65.

64. Tantaleán MV, García L. 1993. Trematodes de la familia Gorgoderidae en anfibios Leptodactylidae de la región altoandina del Perú. Bol Lima 15: 25-27.

65. Tantaleán MV, Sánchez L, Gómez L, Huiza A. 2005. Acantocéfalos del Perú. Rev Peru Biol 12: 83-92.

66. Tkach VV, Lisitsyna OL, Crossley JL, Binh TT, Bush SE. 2013. Morphological and molecular differentiation of two new species of Pseudoacantocephalus Petrochenko, 1958 (Acantocephala: Echinorhynchidae) from amphibians and reptiles in the Philippines, with identification key of the genus. Syst Parasitol 85: 11-26. doi: 10.1007/s11230013-9409-8

67. Tkach VV, Kuzmin Y, Snyder SD. 2014. Molecular insight into systematics, host associations, life cycles and geographic distribution of the nematode family Rhabdiasidae. Int J Parasitol 44: 273-284. doi: 10.1016/j.ijpara. 2013.12. 005

68. Urra F. 2013. Síntesis del conocimiento actual sobre los sapos Rhinella atacamensis, $R$. arunco y $R$. spinulosa. La Chiricoca 16: 4-15.

69. Zar JH. 1996. Biostatistical analysis. $3^{\text {th }}$ ed. New Jersey: Prentice-Hall. 662 p. 